\title{
Absorption of Radiant Energy by Solid Particles in Suspension
}

\author{
James E. Stewart
}

\begin{abstract}
A simple theory is developed to describe the absorption of radiant energy by particles suspended in a transparent medium and also by an absorbing medium containing transparent suspended particles. It is found that in both cases transmittance increases as particle size increases. Apparent deviations from Beer's law and other effects that might introduce difficulties in experimental work are discussed.
\end{abstract}

\section{Introduction}

Radiation-absorption measurements have long been used for quantitative analysis of materials in the gas and liquid phases. Solid materials, however, have been troublesome even for qualitative determinations because of the inherent difficulties in preparing specimens in a reproducible form. Until recently the standard method for study of solids has been to suspend finely divided particles in a medium such as mineral oil in order to reduce the amount of energy lost by scattering. In principle, the concentration of such a mixture, or mull, can be controlled by careful weighing, and the thickness of the mixture can be kept fixed by proper cell design [1]. ${ }^{1}$ But the method is inconvenient, and its usefulness suffers from interference by absorption bands of the suspension medium.

Recently a new technique [2, 3] has been proposed in which finely ground particles of a solid sample are mixed with an alkali halide powder, usually potassium bromide, placed in a die, and pressed until a sintered disk is formed. At the Bureau, small disks, $9.5 \mathrm{~mm}$ in diameter and approximately $100 \mathrm{mg}$ in weight, are produced in a simple die patterned after one described by Anderson [4]. Pressure is applied with an ordinary shop vise. No special precautions are taken for drying the potassium bromide or evacuating the system while pressing. Satisfactory qualitative infrared spectra have been obtained with these pellets, and the method seems promising for quantitative measurements as well.

The purpose of this report is to present a simple theoretical derivation of an absorption law for these disks and to infer from the law certain precautions to be taken when preparing samples for quantitative measurements. The discussion applies equally well to powders suspended in liquid media. Mathematical descriptions of radiation scattered from spherical particles have long been known. But they are quite complicated and cannot be approximated satisfactorily when the particles are about equal in size to the wavelength of incident radiation. In addition, index of refraction curves in the neighborhood and interior of infrared absorption bands are not known for many covalent compounds. A few published dispersion curves $[5,6]$ show that over many infrared absorption bands refractive index

\footnotetext{
${ }_{1}^{1}$ Figures in brackets indicate the literature references at the end of this paper.
}

changes very little. Price and Tetlow [7] have made the same observation from their work on Christiansen windows in infrared spectra. Accordingly, the present study is concerned with the case of no interface refraction, requiring that a perfect index of refraction match between particle and surrounding medium be assumed. In reality this condition can be only approximately true at best.

\section{Derivation of an Absorption Law}

Consider first a single particle of material with absorption coefficient $a$ defined by Beer's law, $I / I_{0}=\exp (-a x)$ for a uniform layer of thickness $x$ (see fig. 1). $d \sigma_{1}$ is an increment of the area $s_{1}$ on which the incident plane wave falls with irradiance ${ }^{2} I_{0}, n$ is a unit vector normal to the particle surface, $j$ is a unit vector perpendicular to the radiation wave front such that the wave travels in the direction, and $d E$ is the energy leaving the particle through $d \sigma_{2}$. Beer's law can be written for a cylindrical increment of volume

$$
d E=I_{0} n \cdot j d \sigma_{1} \exp \left(y_{2}-y_{1}\right) a .
$$

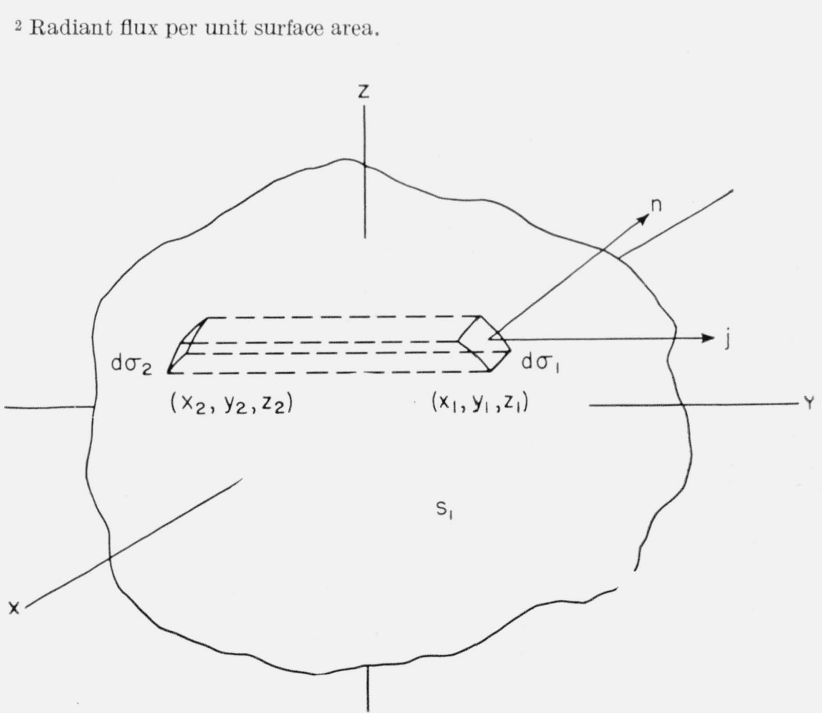

Figure 1. Particle in radiant-energy field, the energy being propagated in the $-j$ direction. 
The projected cross-sectional area of the particle on the plane of the wave front is

$$
A=\int_{s_{1}} n \cdot j d \sigma_{1}
$$

The average transmittance of a single particle is then

$$
\left(I / I_{0}\right)_{p}=\frac{1}{A I_{0}} \int_{S_{1}} d E .
$$

For spherical particles of radius $r$ the integrations in (2) are easily done to give the equivalent expressions

$$
\begin{aligned}
\left(I / I_{0}\right)_{\text {sphere }} & =[1-(2 a r+1) \exp (-2 a r)] / 2 a^{2} r^{2} \\
& =[\exp (2 a r)-2 a r-1] \exp (-2 a r) / 2 a^{2} r^{2} \\
& =2 \sum_{n=0}^{\infty} \frac{(2 a r)^{n}}{(n+2) !} \exp (-2 a r) \\
& =f(r, a) \exp (-2 a r) .
\end{aligned}
$$

Jones [8] has investigated the effect produced in the apparent absorption coefficient by holes in a layer of absorbing substance. The next step in the present development follows his method. Consider a disk containing masses $m_{p}$ and $m_{k}$ of sample and supporting medium of densities $\rho_{p}$ and $\rho_{k}$, respectively. The sample particles are assumed to be spheres of radius $r$, and to be distributed uniformly throughout the unpressed disk on a cubic lattice of constant $\lambda$. The distance between layers of particles parallel to the wave front will be reduced by the pressing. It will be evident from what follows that the distribution of particles within layers can be random so long as the number of particles per layer is constant. In fact, randomness within layers will improve the validity of an approximation which will be made. The volume of disk occupied by sample and by the suspending substance is $v_{p}=m_{p} / \rho_{p}$ and $v_{k}=m_{k} / \rho_{k}$, respectively. The number of particles in the disk is

$$
n_{p}=v_{p} / \frac{4}{3} \pi r^{3}=3 m_{p} / 4 \pi r^{3} \rho_{p},
$$

and the mean distance between particle centers is $\lambda=\left[\left(v_{k}+v_{p}\right) / n_{p}\right]^{\frac{1}{3}}$. Consider first one layer of particles in cells of side $\lambda$. The ratio of the cross-sectional area, $\sigma_{p}$, of the sample particle contained in a single cubic unit to the cross-sectional area, $\sigma_{c}$, of the lattice unit is

$$
\frac{\sigma_{p}}{\sigma_{c}}=\frac{\pi r^{2}}{\lambda^{2}}=\left[\frac{3 \sqrt{\pi} m_{p} \rho_{k}}{4\left(m_{k} \rho_{p}+m_{p}\right)_{k}}\right]^{2 / 3},
$$

and the transmittance for one layer is given by

$$
\left(I / I_{0}\right)_{1}=\left(1-\frac{\sigma_{p}}{\sigma_{c}}\right)+\frac{\sigma_{p}}{\sigma_{c}} f(r, a) \exp (-2 a r) .
$$

Now the number of layers in the disk is $N=\sigma_{c} n_{p} / S$, where $S$ is the disk cross-sectional area. Substituting from above,

$$
N=\frac{1}{r S}\left[\frac{3 m_{p}}{4 \pi \rho_{p}}\left(\frac{m_{k}}{\rho_{k}}+\frac{m_{p}}{\rho_{p}}\right)^{2}\right]^{1 / 3} .
$$

Equation (5) gives the average transmittance of one layer of particles. We assume that the $I$ given by (5) for one layer is the $I_{0}$ for the next layer. The irradiance after the radiation has traversed $N$ layers is therefore

$$
I / I_{0}=\left[\left(1-\frac{\sigma_{p}}{\sigma_{c}}\right)+\frac{\sigma_{p}}{\sigma_{c}} f(r, a) \exp (-2 a r)\right]^{N} .
$$

After the work reported here was completed, a similar discussion appeared in the literature [9], containing an expression for the transmittance of a potassium bromide pellet in the form $I / I_{0}=e^{-a(1-F)}$, in which $F$ is the average transmittance of a single particle and $a$ contains mass, density, and dimension parameters. A comparison of this expression expanded in a Maclaurin's series with eq (7) expanded in a binomial series demonstrates the equivalence of the equations, at least for small particles.

Now let us turn to a similar problem in which the particles are transparent and the suspension medium absorbs energy. A situation such as this is encountered in conventional mulling techniques of sample preparation but will not arise for pellets unless it becomes necessary to use suspension powders that have absorption bands. Let $\alpha$ be the absorption coefficient of the suspension medium and all other parameters remain as defined previously. Proceeding as above, the average transmittance for a single lattice unit is

$$
\begin{aligned}
\left(I / I_{0}\right)_{c} & =\frac{1}{\lambda^{2}}\left\{\int_{S_{1}} \int e^{-\alpha(\lambda-2 r n \cdot j)} n \cdot j d \sigma_{1}+\left(\lambda^{2}-\pi r^{2}\right) e^{-\alpha \lambda}\right\} \\
& =e^{-\alpha \lambda}\left\{\left(1-\frac{\sigma_{p}}{\sigma_{c}}\right)+\frac{\sigma_{p}}{\sigma_{c}} \frac{e^{2 r \alpha}(2 r \alpha-1)+1}{2 \alpha^{2} r^{2}}\right\}
\end{aligned}
$$

The transmittance for the first layer of cells equals the same value and, making the same assumption used earlier regarding the average $I_{0}$ for the next layer, the transmittance of the specimen is

$$
I / I_{0}=\left(I / I_{0}\right)_{c}^{N}
$$

\section{Discussion}

It is not clear how eq (2) might be generalized further in order to demonstrate the effect of particle shape. ${ }^{3}$ However, some easily integrable shapes can be investigated. For example, a disk-shaped particle with volume and cross-sectional area equal to that of a sphere with radius $r$ must have thickness $4 r / 3$. With its flat surface parallel to the radiation wave

${ }^{3}$ In this connection, note that $\nabla \cdot \exp a\left(y_{2}-y_{1}\right) j \equiv \nabla \cdot \exp \left(-a_{\eta}\right) j=\frac{\partial}{\partial \eta} \exp \left(-a_{\eta}\right) \neq 0$, so the integrand in (2) cannot be the curl of any function and Gauss's theorem cannot be applied. 


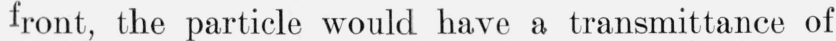
$\left(I / I_{0}\right)_{\text {disk }}=\exp (-4 a r / 3)$. For the sphere $\left(I / I_{0}\right)_{\text {sphere }}$ $=\exp (-2 a r)$ for sufficiently small $a r$.

Equation (7) for spherical particles involves the radius in a complicated manner. If ar is sufficiently small so that terms beyond those of first degree in the expansions of $\exp (2 a r)$ and $\exp (-2 a r)$ can be discarded, the expression becomes

$$
\begin{aligned}
\ln I / I_{0} & \approx N \ln \left[\left(1-\frac{\sigma_{p}}{\sigma_{c}}\right)-\frac{\sigma_{p}}{\sigma_{c}}\left(1+\frac{2 a r}{3}\right)(1-2 a r)\right] \\
& \approx N \ln \left(1-\frac{\sigma_{p}}{\sigma_{c}} \frac{4 a r}{3}\right) .
\end{aligned}
$$

We may now expand this expression in a Maclaurin's series, keeping only the leading term, substitute (4) and (6), and make the simplifying assumption $m_{p} \ll m_{k}$ (which is true in practice). The result is

$$
\lim _{r \rightarrow 0} \ln I / I_{0}=-a m_{p} / S \rho_{p} .
$$

This is recognized as Beer's law for a mass $m_{p}$ of sample in the form of a homogeneous condensed film. For larger particles the sensitivity of (7) to changes in $r$ can best be demonstrated by assuming some typical values for the other parameters and plotting $I / I_{0}$ versus $r$ (see fig. 2). There is indeed a significant dependence on particle size, which is most serious for bands with highest absorption coefficient. As these are the bands most likely to be used for quantitative purposes, the necessity for controlling particle size is clear. It is also apparent that band shape should be affected by particle size. The curves of figure 2 predict that a sample prepared with large particles will have a higher apparent transmittance than a sample with the same mass of small particles. This applies also to specimens containing clusters of small particles. In this case $r$ must be taken as the cluster radius.

It has been observed experimentally that absorption bands do become more intense as particle size is reduced. Some observations on polyvinyl chloride have been made. It is recognized that scatter plays an undetermined role in these measurements. However, it is not likely that reduction of particle size will result consistently in greater scatter at an absorption band, and hence in greater apparent absorption. Furthermore, no asymmetric variations in band shapes, such as Christiansen windows, were observed.

Calculations from eq (7), using typical values ${ }^{4}$ for the parameters, provided the encouraging result that the apparent transmittance is independent of $m_{k}$ within the practical measuring limits of modern spectrophotometers. In order to verify this experimentally, two pellets were prepared containing the same quantity $(2.6 \mathrm{mg})$ of stearic acid but different amounts of potassium bromide $(42.25 \mathrm{mg}$

\footnotetext{
${ }^{4} m_{p}=2 \mathrm{mg}, \rho_{p}=1 \mathrm{~g} / \mathrm{cm}^{3}, \rho_{k}=0.2 \mathrm{~g} / \mathrm{cm}^{3}, S=0.714 \mathrm{~cm}^{2}, a=100$ and $200 \mathrm{~cm}^{-1}$, and $m_{\text {k }}$ varied between 50 and $250 \mathrm{mg}$.
}

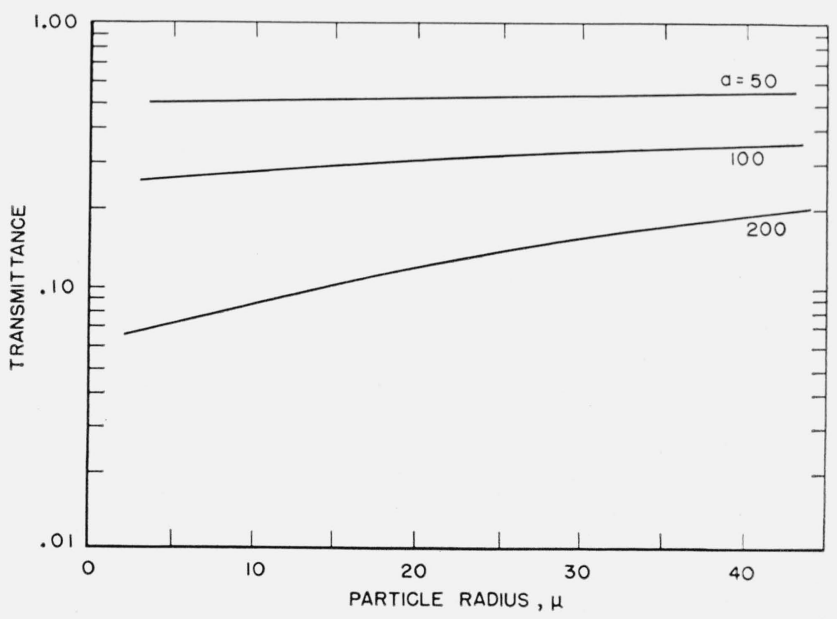

FIGURE 2. Effect of radius of particles with various absorption coefficients on transmittance.

Curves are calculated from eq (7), using the following values: $m_{p}=0.01 \mathrm{~g}$; $m_{k}=0.224 \mathrm{~g} ; \rho_{p}=1 \mathrm{~g} ; \rho_{k}=0.2 \mathrm{~g} / \mathrm{cm}^{3} ; S=0.714 \mathrm{~cm}^{2} ; a=50,100$, and $200 \mathrm{~cm}^{-1}$

and $294.85 \mathrm{mg}$ ). Transmittance was measured relative to base lines drawn through maxima adjacent to the minimum in question. The transmittances of corresponding bands at wavelengths greater than $9.1 \mu$ were equal for the two pellets within 2 or 3 percent. In the range 3 to $9.1 \mu$ bands were either too nearly opaque or were too close together to permit reasonable base lines to be drawn. The combination bands near $2.4 \mu$ had transmittances of 78.7 and 86.1 percent for the pellet containing more and less potassium bromide, respectively.

In the case of alkali halide pellets the distribution of sample particles in the mixture is determined by the density of the suspending medium before pressing, that is, by the volume of material plus included air spaces. But if $m_{p} \ll m_{k}$ then $\rho_{k}$ appears in (7) only in factors $m_{k} / \rho_{k}$ so transmittance should be practically independent of $\rho_{k}$ also. In the case of powder suspended in liquid the question of which value to use for $\rho_{k}$ does not arise.

Figure 3 shows calculated curves plotted on semilogarithmic coordinates of $I_{0} / I$ versus $m_{p}$ for a condensed homogeneous film and for suspensions made with three different particle sizes. It is seen that for samples prepared from powders there are apparent "deviations from Beer's law" in the sense that straight lines are not obtained. The deviations are not great for small radii; however, it is seen again that apparent absorption is greatly reduced for larger particles or clusters.

Because there is a finite probability that radiant energy will traverse a pellet without encountering a sample particle, it is expected that the transmission for opaque particles will be somewhat greater than the instrumental transmission zero determined with an opaque shutter in the optical path. This is equivalent to stating that $I / I_{0}$ will not become zero as a approaches infinity in eq (7). Calculations using eq (7) with the parameters $a=\infty, \rho_{p}=1 \mathrm{~g} / \mathrm{cm}^{3}$, $\rho_{k}=0.2 \mathrm{~g} / \mathrm{cm}^{3}, S=0.714 \mathrm{~cm}^{2} m_{p}=0.01 \mathrm{~g}$, and $m_{k}=$ 


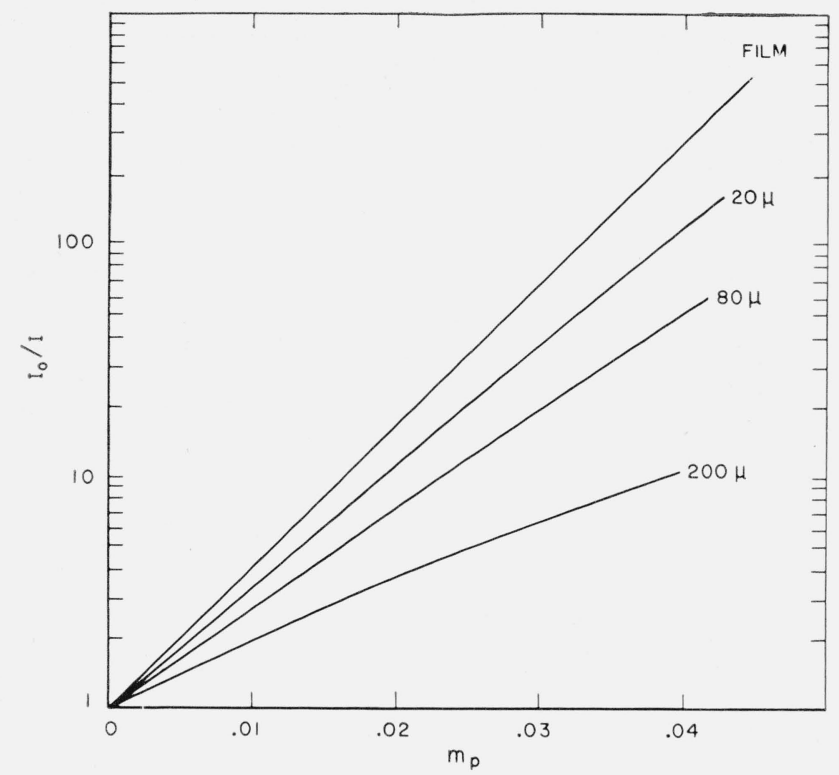

FiguRE 3. Effect of radius of particles and mass of sample on absorbance.

Curves are calculated from eq (7), using the following values: $a=100 \mathrm{~cm}^{-1}$; $m_{k}=0.224 \mathrm{~g} ; \rho_{p}=1 \mathrm{~g} / \mathrm{cm}^{3} ; \rho_{k}=0.2 \mathrm{~g} / \mathrm{cm}^{3} ; S=0.714 \mathrm{~cm}^{2}$.

$0.224 \mathrm{~g}$ predict that for particles up to about $47 \mu$ in diameter the transmission zero will be raised less than 1 percent, for $72 \mu$ diameter particles the zero will be raised to 5 percent transmission, and for $200 \mu$ diameter particles the zero will be raised to 34 percent transmission.

Now the sample is to be considered as a multicomponent system. If the components are distributed uniformly throughout the particle, it is sufficient to define $a$ in (3) by

$$
a=\sum_{i} a_{i} c_{i}
$$

in which $a_{i}$ and $c_{i}$ are the absorption coefficient and molar concentration of the $i$ th component. 'This reduces to $a=a_{i} c_{i}$ for a frequency chosen so that $a_{j}=0$ for $j \neq i$. It is sufficient then to discuss (7) relative to changes in $a$. Figure 4 contains a plot of $I_{0} / I$ versus $a=a_{i} c_{i}$ for two particle sizes and a condensed homogeneous film with arbitrary values assumed for parameters $m_{p}, m_{k}, \rho_{p}, \rho_{k}$, and $S$. Deviations from linearity are serious for very large radii but can probably be disregarded for small particles uniformly distributed in the suspension. The deviations are seen to be less serious for small $a$. If the components are not uniformly distributed throughout the individual particles, the calculations are more difficult. An example of such a situation might occur when material undergoes chemical changes while in the form of particles. In degradation studies, for example, degradation products might be found more concentrated at the surface of the particles than in the interior.

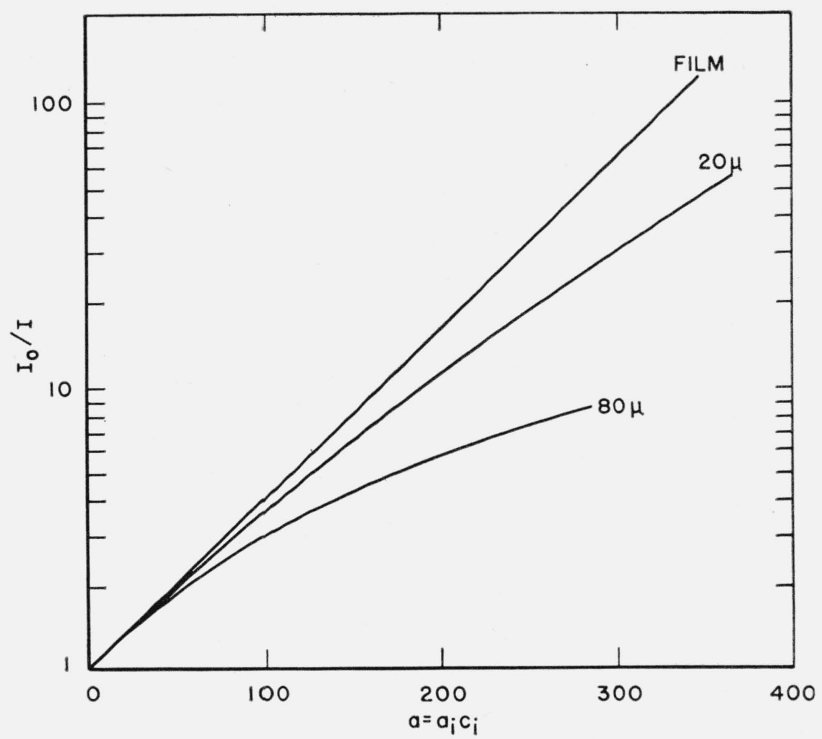

FIGURE 4. Effect of absorption coefficient and radius of particles in multicomponent system on absorbance.

Curves are calculated from eq (7), using the following values: $m_{p}=0.01 \mathrm{~g}$, $m_{k}=0.224 \mathrm{~g} ; \rho_{p}=1 \mathrm{~g} / \mathrm{cm}^{3} ; \rho_{k}=0.2 \mathrm{~g} / \mathrm{cm}^{3} ; S=0.714 \mathrm{~cm}^{2}$.

If $a$ is a function of $p$, the distance from the particle center, then Beer's law for a cylindrical increment of volume with radius $z$ is

$$
\frac{d I}{I}=-a(p) d x, \quad \frac{I}{I_{0}}=\exp \left[-\int_{x_{1}(z)}^{x_{2}(z)} a(p) d x\right] .
$$

The average transmittance of the sphere is

$$
\left(I / I_{0}\right)_{p}=2 \pi I_{0} \int_{0}^{r} \exp \left[-\int_{x_{1}(z)}^{x_{2}(z)} a(p) d x\right] z d z / \pi r^{2} I_{0} .
$$

The integration is not a convenient one even for simple forms of $a(p)$. It will not be discussed further except to stress that it is dependent on the functional form of $a$. It is therefore not legitimate to assume a linear "Beer's law plot" for solid particles undergoing chemical change.

We turn now to the case of transparent particles suspended in an absorbing medium. 'This situation occurs frequently when mulls are prepared with mineral oil, for example. It also occurs when the pellet technique is used with potassium bromide that contains some water. The limiting behavior of eq (9) for vanishing particle size may be seen by noting that $\left[e^{2 r \alpha}(2 r \alpha-1)+1\right] / 2 \alpha^{2} r^{2}$ approaches $e^{2 r \alpha}$ asymptotically as $r$ approaches zero. Further substitution of two terms of a power series for the exponentials leads to the expression

$$
\lim _{r \rightarrow 0} I / I_{0}=\exp \left[-\alpha\left(t-\frac{3}{2} t_{p}\right)\right]
$$

in which $t$ is the thickness of the suspension and $t_{p}$ 


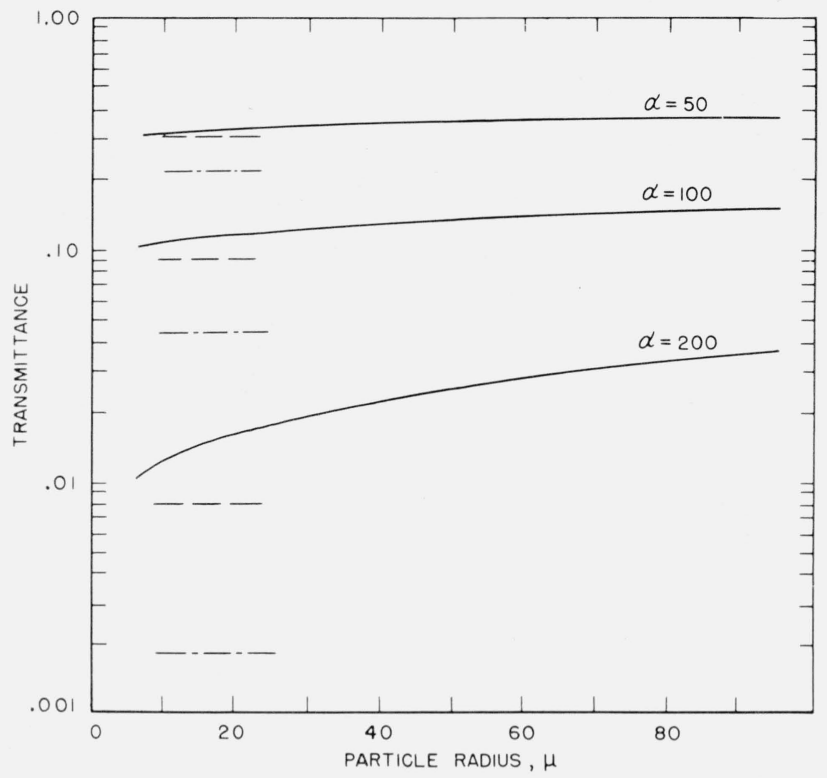

FIGURE 5. Effect of radius of transparent particles in media with various absorption coefficients on transmitance.

Curves are calculated from eq (9), using the following values: $m_{m}=22.4 \mathrm{mg}$ $m_{p}=10 \mathrm{mg} ; \rho_{p}=\rho_{m}=1 \mathrm{~g} / \mathrm{cm}^{3} ; S=0.714 \mathrm{~cm}^{2}$. The dash lines represent transmittance in the limit of vanishing particle radius and the dash-dot-dash lines represent transmittance of pure material containing no particles for the corresponding absorption coefficients.

is the thickness that the powder alone would have if it were in the form of a condesned homogeneous film. The occurrence of the factor $3 / 2$ in the exponent is surprising. Of course as $r$ approaches zero it is no longer reasonable to integrate Beer's law over a sphere, as was done in deriving these equations. Values calculated from eq (9), using assumed parameters, are plotted versus particle size in figure 5. In general, samples containing the same amounts of material transmit more energy as the size of the suspended particles increases. The increase will occur at a greater rate for bands of higher absorption coefficient. The change in appearance of a hypothetical absorption band as particle size is varied is demonstrated in figure 6 . The envelope labelled "ideal" represents liquid containing no powder as given by eq (10) without the factor $3 / 2$.

While obtaining spectra of suspensions of polyvinyl chloride in liquid media using a doublebeam spectrometer, it was observed that complete compensation for bands arising from the liquid could not be obtained. For example, if a point on each side of the band were compensated exactly by adjusting a variable thickness cell, then that portion of the band falling between these points would be overcompensated and other portions would be undercompensated. Furthermore, when a quantity of compensating liquid calculated on an "ideal" basis (i. e., from eq (10) without the factor $3 / 2$ ) was placed in the reference beam, bands were strongly overcompensated. Scatter alone would not be expected to produce these symmetric changes in band shape. However, the present theory involving

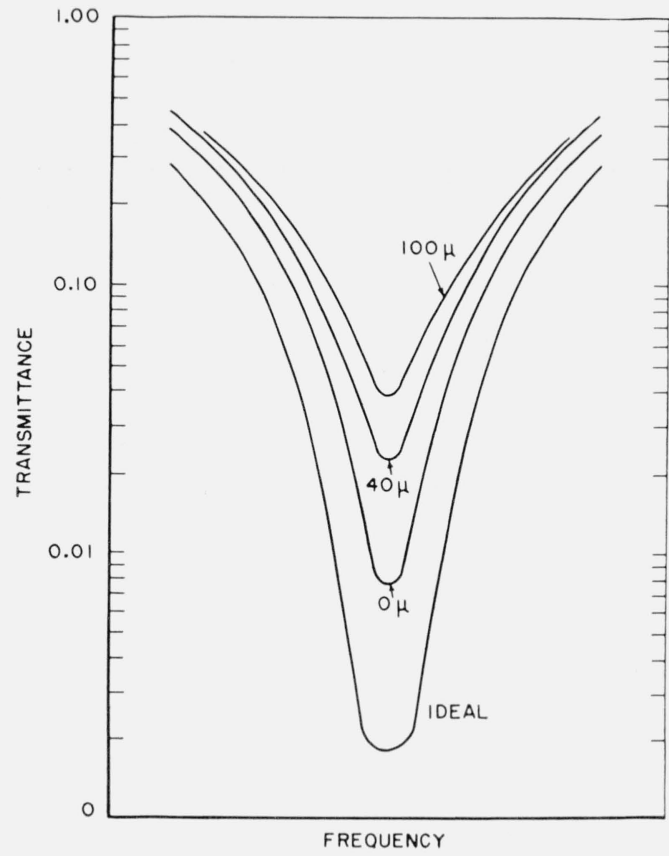

FigURE 6. Effect of size of transparent particles on the shape of the absorption bands of an absorbing materialjin which the particles are dispersed.

The curves were drawn from the data in figure 5 .

absorption alone gives a resonable qualitative description. For if an ideal-band envelope is calculated by using a thickness appropriate for exact compensation at points on the side of the "suspension" band, it will have greater and less transmittance than the suspension band for frequencies inside and outside these points, respectively. The difference between the two contours will produce a differential band similar to the ones observed. Attempts at compensation would be expected to be more successful with small particles.

\section{Summary}

A simple but plausible model of solid particles suspended in a transparent medium, with the postulate that refractive index changes over molecularvibration absorption bands are sufficiently weak to be ignored, has yielded the following predictions:

1. The apparent absorption is dependent on particle shape.

2. The apparent absorption is dependent on particle size. Samples containing large particles transmit more energy than samples with the same mass of small particles. Absorbance as a function of mass of sample for a one component system deviates from linearity most markedly when large particles are used. For smaller particles the absorbance curve becomes linear and in fact coincides with the Beer's law plot obeyed by a condensed homogeneous film of the same material. In multicomponent systems the form of $I / I_{0}$ depends upon the way in which the components are distributed in the par- 
ticles. In the case of uniform distribution within the particles, deviations from linearity in absorbance versus concentration are greatest for large particles, and again absorbance approaches that of a condensed homogeneous film for small particles. All the remarks made here regarding particle size apply to cluster size in the case of incompletely dispersed systems. The desirability of obtaining small particles and the necessity for controlling particle size distribution and for producing complete dispersion of particles in the suspension medium are clear.

3. The apparent absorption is not greatly affected by variation in mass and density of suspending material.

4. In addition, a related model of transparent particles in absorbing media has led to the conclusion that apparent transmittance of a suspending medium should increase as the size of the particles which it contains increases.

The author is grateful for discussions with Mary Reiney Harvey, F. A. Smith, and E. Carroll Creitz during the development of experimental technique. Special thanks are due D. H. Anderson for providing details of his pellet-pressing method before publication.

\section{References}

[1] J. E. Ard, Anal. (hem. 23, 680 (1951).

[2] U. Schiedt, Z. Naturforsch. 7b, 270 (1952); 8b, 66 (1953). See also H. Hausdorff, Appl. Spectroscopy [2], 7, 75 (1953) for a summary and some additiona! results.

[3] M. M. Stimson and M. J. O'Donnell, J. Am. Chem. Soc. \%4, 1805 (1952).

[4] D. H. Anderson and N. B. Woodall, Anal. Chem. 25, 1906 (1953).

[5] A. H. Pfund, J. Opt. Soc. Am. 25, 351 (1935).

[6] M. A. Pittman, J. Opt. Soc. Am. 29, 358 (1939).

[7] W. C. Price and K. S. Tetlow, J. Chem. Phys, 16, 1157 (1948).

[8] R. N. Jones, J. Am. Chem. Soc. 74, 2681 (1952).

[9] W. R. Harp, Jr., H. Stone, and J. W. Otvos, Anal. Chem. 26, 431 (1954). Presented at the Pittsburgh conference on Analytical Chemistry and Applied Spectroscopy (March i 954 ).

Washington, May 3, 1954. 